\title{
The Proposed Fuzzy_IAMR Approach
}

\author{
Ouarda Hachour \\ Institute of Electrical and Electronics Engineering, Boumerdes University, Boumerdes, Algeria. \\ Email: hachour_ouarda@yahoo.fr
}

Received November $28^{\text {th }}, 2012$; revised December $30^{\text {th }}, 2012$; accepted January $12^{\text {th }}, 2013$

\begin{abstract}
In this paper we present a fuzzy_IAMR Intelligent Autonomous Mobile Robot navigation approach of Autonomous Mobile Robot. The robot has to find a collision-free trajectory between the starting configuration and the goal configuration in a static unknown environment containing some obstacles. To deal with autonomy requirements and to present a real intelligent task, the use of the Fuzzy Logic FL has an advantage of adaptivity such that this approach works perfectly even if an environment is unknown. In this context, we present a software implementation Fuzzy Logic FL path planning in a terrain. Fuzzy logic allows a continuum of control variables such as heading angles and speeds to be considered, as opposed to the discrete numbers used in crisp behaviors. Artificial intelligence, including Fuzzy logic has been actively studied and applied to domains such as automatically control of complex systems like robot. In $\mathrm{f}$ act, recognition, learning, decision-making, and action constitute the principal obstacle avoidance problems, so it is interesting to replace the classical approaches by technical approaches based on intelligent computing technologies. This technology FL is becoming useful as alternate approach to the classical techniques one. Also, fuzzy logic can be viewed as an attempt to bring together conventional precise mathematics and humanlike decision-making concepts. Fuzzy logic can be a valid approach solving control problem in a wide range of applications. To deal with the principle, the robot moves within the unknown environment by sensing and avoiding the obstacles coming across its way towards the unknown target. This algorithm provides the robot the possibility to move from the initial position to the final position (target) without collisions where the main factors of moving are included such as learning, deciding, acting, cognition, perception, and thinking. The robot succeeds to reach the target without collisions. The results gotten of the FL on randomly generated terrains are very satisfactory and promising. The extension of the FL for solving both paths planning and trajectory planning.
\end{abstract}

Keywords: Intelligent Autonomous Mobile Robot (IAMR); Expert System; Fuzzy Logic (FL); Navigation

\section{Introduction}

The autonomous robot navigation problem has been studied thoroughly by the robotics research community over the last years. The basic feature of an autonomous mobile robot is its capability to operate independently in unknown or partially known environments.

The autonomy implies that the robot is capable of reacting to static obstacles and unpredictable dynamic events that may impede the successful execution of a task. To achieve this level of robustness, methods need to be developed to provide solutions to localization, map building, planning and control. The robot has to find a collision-free trajectory between the starting configuration and the goal configuration in a static or dynamic environment containing some obstacles.

Autonomous mobile robots in unstructured complex environments, densely cluttered with obstacles is still a major challenge in goal-directed robotic vehicle applications. The problem is particularly difficult because some of the navigational objectives may be in opposition to one another, to see the main composite of navigation system. It is important that algorithms for navigation control in cluttered environments not be too computationally expensive as this would result in a sluggish response.

The control complexity is overcome by decomposing the navigation control problem into more simple and well-defined sub-problems that can be controlled independently and in parallel. These sub-problems and their controllers are known as reactive behaviors, and this approach is known as behavior robotics. It has attracted the interests of many robotic applications and has even been used in industrial process control applications [1,2].

Autonomous robots which work without human operators are required in robotic fields. In order to achieve tasks, autonomous robots have to be intelligent and should decide their own action. The behavior robotics has grown quickly resulting in the development of reactive fuzzy behavior methods that use fuzzy logic control- 
lers, which can handle uncertainty in the robot information.

Fuzzy logic also allows a continuum of control variables such as heading angles and speeds to be considered, as opposed to the discrete numbers used in crisp behaviors. In addition, it allows the navigation algorithm to be programmed using linguistic terms, which is the way a designer naturally thinks.

Probably, the greatest strength of behavior-based fuzzy approaches is that they operate on and reason with uncertain perception based information, which makes them suitable even for difficult environments such as unknown terrains.

The concept of behavior control was initially seen as a special form of decentralized switching control in which each behavior is fully autonomous, and when allowed, can control the robot on its own without regard to other behaviors. Under what we will call the standard behavior paradigm, each behaviors triggers a single control command that best meets the control responsibilities specific to that behavior $[3,4]$.

A lot of versions have been proposed for the concept of fuzzy system these efforts have been based on developing fuzzy versions of the standard behavior structure in which each behavior chooses one action out of the possible actions, in this case a fuzzy action.

These structures were found to have performance problems especially since they treat behaviors as fully autonomous, which tend to cause the robot to be indecisive when the behaviors have mutually exclusive interests with nearly equal importance.

This observation led to the introduction of what we will collectively refer to as preference-based behavior systems. The history of autonomous mobile robotics research has largely been a story of closely supervised, isolated experiments on platforms which do not last long beyond the end of the experiment. There is no universally accepted definition of the term robot.

Typical definitions encompass notion of mobility, programmability, and the use sensory feedback in determining subsequent behavior.

When the autonomous robot decides its action, it is necessary to plan optimally depending on their tasks.

More, it is necessary to plan a collision free path minimizing a cost such as time, energy and distance.

When an autonomous robot moves from a point to a target point in its given environment, it is necessary to plan an optimal or feasible path avoiding obstacles in its way and answer to some criterion of autonomy requirements such as: thermal, energy, time, and safety for example $[5,6]$.

This paper deals with the intelligent path planning of AMR Autonomous Mobile Robot in an unknown environment. The aim of this paper is to develop a Fuzzy logic IAMR Intelligent Autonomous Mobile Robot algorithm for the IAMR stationary obstacle avoidance to provide them more autonomy and intelligence.

Today, researchers have at their disposal, the required hardware, software, and sensor technologies to build Intelligent Autonomous vehicles IAV. More, they are also in possession of a computational tool such as FL that is more effective in the design and development of IAV than the predicate logic based methods of traditional Artificial Intelligence.

For example, Fuzzy Logic FL and Expert System ES are well established as useful technologies that complement each other in powerful hybrid system.

Hybrid intelligent systems are now part of the repertoire of computer systems developers and important research mechanisms in the study of Artificial Intelligent $[7,8]$.

The integration of ES and FL has proven to be a way to develop useful real-world applications, and hybrid systems involving robust adaptive control. Robots are distributed systems; multiple sensory, reasoning, and motor control processes run in parallel, often on separate processor hate rate only loosely coupled with one another. Each of these procure necessarily maintains its own separate, limited representation of the world and task; requiring them to constantly synchronize with the central knowledge base is probably unrealistic.

Automated reasoning systems are typically built on a transaction-oriented model of computation. Knowledge of the world is stored in a database of assertion in some logical language, indexed perhaps by predicate name.

The motion of mobile robots in an environment where there are stationary obstacles, and other moving objects, requires the existence of algorithms that are able to solve the path and motion planning problem of these robots so that collisions are avoided.

On the other hand, a suitable control law has to be designed, in order for the mobile robot to execute the desired motion. The problem becomes more difficult when the parameters that describe the model and/or the workspace of the robot are not exactly known.

In order to achieve the necessary tasks, autonomous robots to be intelligent and should decide on their own action. When the autonomous robots decide its action, it is necessary to plan optimally depending on their asked missions. In the case of a mobile robot, it is necessary to plan a collision-free path minimizing a cost such a time and distance.

In particular the robot science has shown the capabilities of robots including: power consumption, autonomous reaction capabilities, design complexity, learning, own decision, mission flexibilities, performing missing and reacting are done in efficient manner to deal with anthropomorphist principle. This is was a dream in all Arti- 
ficial Intelligence world realizations.

This paper deals with the intelligent fuzzy controller for autonomous mobile robots moving with stationary obstacles to provide them more autonomy and intelligence.

Artificial intelligence, including Fuzzy logic has been actively studied and applied to domains such as automatically control of complex systems like robot.

In fact, recognition, learning, decision-making, and action constitute the principal obstacle avoidance problems, so it is interesting to replace the classical approaches by technical approaches based on intelligent computing technologies. This technologies FL is becoming useful as alternate approaches to the classical techniques one.

The proposed approach can deal a wide number of environments. This system constitutes the knowledge bases of FL approach allowing recognizing the fuzzy situation of the target localization and obstacle avoidance, respectively. This approach can be realized in efficient manner and has proved to be superior to combinatorial optimization techniques, due to the problem complexity.

\section{Path Planning}

To detect all possible obstacles, the robot is supposed to have vision system (camera). To operate in certain dynamic environments, the use of two or more sensors can guarantee to deliver acceptably accurate information all of he time. Thus the redundancy can be useful for autonomous systems as in the human sensory system.

When an autonomous robot moves from a point to a target point in it given environment it is necessary to plan an optimal or feasible path avoiding obstruction in its way and answering to autonomy requirements such as: thermal, energy, Communication Management, Mechanical design, etc.

To evaluate the performances of vehicles one must answer to all factors to be embedded with robot when it executes its mission, this is summarized in how to perform all tasks, such as: Intelligence, Navigation, reliability, reliability, and safety.

In order to make use of environmental models mobile robots always must know their current position and orientation in their environment. Therefore, the ability of estimating their position and orientation is one of the basic preconditions for the autonomy of mobile robots.

\subsection{Mapping and Cognition of Maintaining the Integrity of the Environment}

The use of map to structure our environment has often been more efficient than previous technique. The difficulty in building a map of the environment lies in the cognition representation. For same types of navigation, it is more advantageous to use an implicit one. In the intel- ligent robot behavior, this environment model map has an important role to play.

So, building a map of the sensory input space is more interesting especially we give this subdivision by the following points: geometrical level, topological level and semantic level. For the first one the aim work is focused on the cartographer work where the flat earth model viewable area is an ideal planner surface $S$ defined by the points of contact of the objects projected into the ground. The second illustrates the features map; it consists of the decomposition on free and occupied space, and gives a relationship between the free spaces. In this case, a gridmap is more suitable for the unknown environment. In addition to determine a valid, safe path grid-mapped environments, an intersection of parallel horizontal and vertical lines is the key of digital field analysis, that is the way to interpret safety and danger areas.

In the previous step, the topological level provides the entering of organization map and offers all features associated with the appropriate environment. In this context, the homogenous grid-map obtained must be associated with appropriate level useful in real-time, learning, generalizing, and approaching human-like reasoning for each particular situation, this association is achieved by:

- $\mathrm{P}$ is 1 : if grid is free.

- $\mathrm{P}$ is 0 : if not.

\subsection{Path Panning}

The goal of the navigation process of mobile robots is to move the robot to a named place in a known, unknown or partially known environment. In most practical situations, the mobile robot cannot take the most direct. Path from the start to the goal point. So, path planning techniques must be used in this situation, and the simplified kinds of planning mission involve going from the start point to the goal point while minimizing some cost such as time spent, chance of detection, or fuel consumption. Often, a path is planned off-line for the robot to follow, which can lead the robot to its destination assuming that the environment is perfectly known and stationary and the robot can rack perfectly.

Early path planners were such off-line planners or were only suitable for such off-line planning. However, the limitations of off-line planning led researchers to study on-line planning, which relies on knowledge acquired from sensing the local environment to handle unknown obstacles as the robot traverses the environment One of the key issues in the design of an autonomous robot is navigation, for which, the navigation planning is one of the most vital aspect of an autonomous robot. Therefore, the space and how it is represented play a primary role in any problem solution in the domain of mobile robots because it is essential that the mobile robot 
has the ability to build and use models of its environment that enable it to understand the scene navigation's structure. This is necessary to understand orders, plan and execute paths.

Moreover, when a robot moves in a specific space, it is necessary to select a most reasonable path so as to avoid collisions with obstacles. Several approaches for path planning exist for mobile robots, whose suitability depends on a particular problem in an application.

For example, behavior-based reactive methods are good choice for robust collision avoidance. Path planning in spatial representation often requires the integration of several approaches. This can provide efficient, accurate, and consistent navigation of a mobile robot.

The major task for path-planning for single mobile robot is to search a collision-free path. The work in path planning has led into issues of map representation for a real world. Therefore, this problem considered as one of challenges in the field of mobile robots because of its direct effect for having a simple and computationally efficient path planning strategy [9-14].

The multi-level structure of path planning and execution propounded in provides a basic framework for dealing with problems in the control of autonomous vehicles. There are three basic levels of path planning and execution.

In global path planning, prior knowledge of the workspace is available which means that the robot navigates in known environment. Local path planning methods use ultrasonic sensors, laser range finders, and on-board vision systems to perceive the environment to perform on-line planning in this case a prior knowledge of the environment is not necessary and the robot navigate in an unknown environment.

\subsection{Robot Control}

Traditionally, motion planning and control have been separate fields within robotics. However, this historical distinction is at best arbitrary and at worst harmful to the development of practically successful algorithms for generating robotic motion. It is more useful to see planning and control as existing on the same continuum.

In assistive robotics, a manipulator arm constitutes one possible solution for restoring some manipulation functions to victims of upper limb disabilities. When the robot is far from its objective only the mobile base moves; thus avoiding obstacles if necessary. When the objective is close to the robot, both mobile base and arm move and redundancy can be used to maximize a manipulability criterion. The partial results obtained with the real robot consolidate the results of simulation. The work does not propose an autonomous path planning and navigation of the mobile arm but assistance to the user for remote controlling it.
The control task becomes more complex when the configuration of obstacles is not known a priori. The most popular control methods for such systems are based on reactive local navigation schemes that tightly couple the robot actions to the sensor information. In the next session we discuss fuzzy logic controller principle.

\section{The Proposed Fuzzy_Logic Controller}

Fuzzy Logic FL and Expert System ES are well established as useful technologies that complement each other in powerful hybrid system. Hybrid intelligent systems are now part of the repertoire of computer systems developers and important research mechanisms in the study of Artificial Intelligent. The integration of ES and FL has proven to be a way to develop useful real-world applications, and hybrid systems involving robust adaptive [15, 16].

Because of the environmental uncertainties, fuzzy behavior systems have been proposed by researchers. The most difficult problem in applying fuzzy-reactive behavior based navigation control systems is that of arbitrating or fusing the reactions of the individual behaviors, which is addressed in this work by the use of preference logic $[17,18]$.

It was shown in simulation and experimental results that the proposed method allows the robot to smoothly and effectively navigate through cluttered environments such as dense forests. Experimental comparisons with the vector field histogram method (VFH) show that the proposed method usually produces smoother albeit longer paths to the goal.

The high-level subsystem uses fuzzy logic to design a fusion of sensor data, map building, and path planning tasks. The fuzzy/evidence navigation based on the building of a local map, represented as an occupancy grid. The path planning algorithm is based on a modified potential field method.

In this algorithm, the fuzzy rules for selecting the relevant obstacles for robot motion are introduced. Also, suitable steps are taken to pull the robot out of the local minima. Particular attention is paid to detection of the robot's trapped state and its avoidance. One of the main issues in this case is to reduce the complexity of planning algorithms and minimize the cost of the search.

The performance of the proposed system is investigated using a dynamic model of a mobile robot. Simulation results show a good quality of position tracking capabilities and obstacle avoidance behavior of the mobile robot. The missing point that this work does not deal with it is the navigation of a mobile robot in a dynamic environment.

Fuzzy Logic has long been beset with the often muddied distinction between inferences made in a meta-lan- 
guage from statements in an object-language, on the one hand, and on the other hand, the formation in the object language itself of an implicative combination of its own statements. Both the need for this distinction and the difficulty of keeping to it becomes more accurate in the fuzzy environment.

Fuzzy models can obviously be made to work very well indeed. The big advantage of a fuzzy model is that it is relatively simple to construct and is in itself a simple structure. It does not require the modeler to have a deep mathematical insight, but relies more on intuition and experience of the process. Its greatest value must be, therefore, in those areas where such qualitative process knowledge is predominant and essential for understanding.

The theory has shown that the fuzzy models can be successfully constructed; the overall concept needs a considerably more detailed investigation before its true worth can be evaluated. Many of the successful application of fuzzy logic have shown the importance of rule bas fuzzy control.

Control is meant in the most general sense: it includes actual closed loop control, expert systems, and all kinds of man-machine systems where the decision of the human "component" is supported or modified by the conclusion obtained by the application of approximate reasoning on the set of available rules.

Zadeh's original idea of constructing relations from the rules, and the simplified computational techniques (both the one invented by Mamdani and the methods applied in some of the Japanese applications) can be interpreted as approximate computation of the "graph" expressing the true nature of the system modelled. This "graph" is mapping which maps the fuzzy power set of the input universe to the fuzzy power set of the output universe, where all the rules can be considered as approximate "points" of the mapping.

If the system and the available knowledge are defined, i.e. fuzzy, the expert system must be replaced by a fuzzy rule based control system-this is what was proposed by Zadeh.

The fuzzy rules containing linguistic terms as values for the variables represent fuzzy cases and are typical "fuzzy points" of the graph of R. So it is supposed that the mapping from input to output is inherently fuzzy. If the membership functions in use have necessary single point i.e. cores with cardinality one, the fuzzy mapping defines always a classic mapping from core to core.

This interpretation of the fuzzy mapping points to the importance of the resolution principle. Rules stands for a fuzzy point of the fuzzy mapping, the next step is to clarify how well a set of rules, i.e. a set of fuzzy points can describe the hypothetical mapping. If the analogy is taken from ordinary functions, the curve of an arbitrary function can be approximated better and better, e.g. by a polynomial function, if one and more points of the function are known. In most industrial problems where fuzzy control is applicable, the state variables have interval range, with minimal and maximal possible values and fully ordered structure.

Also, fuzzy logic can be viewed as an attempt to bring together conventional precise mathematics and humanlike decision-making concepts. Fuzzy logic can be a valid approach solving control problem in a wide range of applications. In particular, embedded architectures are likely to use fuzzy logic in the future for dedicated applications.

The Fuzzy Logic Controller FLC can be considered as a system given an input vector computes, and an output vector by a linguistic rule.

To define the complexity of a fuzzy controller we consider some typical parameters such as the number input and the number output, the dimension of the rule base, the number of membership functions per input, the precision and the methods chosen to performed the three well known steps: fuzzification, inference and defuzzification steps. Fuzzy logic is based on the concepts of linguistic variables and fuzzy sets.

The fuzzy logic proves to be a robust tool to solve all one imprecise problem. The three well-known stages used generally are: the fuzzification, the Inference rules and the defuzzification, which are the stages keys to realize the fuzzy principle.

To build intelligent systems that are able to perform complex requiring massively parallel computation, a knowledge of the environment structure and interacting with it involves abstract appreciation of natural concepts related to, the proximity, degree of danger, etc.

The implied natural language is represented through fuzzy sets involving classes with gradually varying transition boundaries. As human reasoning is not based on the classical two-valued logic, this process involves fuzzy truths, fuzzy deduction rules, etc. This is the reason why FL is closer to human thinking and natural language than classical logic. The fuzzy model used in this conception is presented in Figure 1. Figure 2 clarifies the idea of fuzzy linguistic formulation where we propose Robot obstacle avoidance.

\section{Where:}

$A_{i}$ : the direction of the robot. $D_{i}$ : intermediate distance (see the Figure 2). The membership labels for distance $D_{i}$ in the Figure 3 are defined as:

- SP: Start position.

- MP: Middle position.

- VP: Visual position.

The membership functions of direction $A_{i}$ are presented in Figure 4, where fuzzy labels are defined as: 




Figure 1. Fuzzy model.

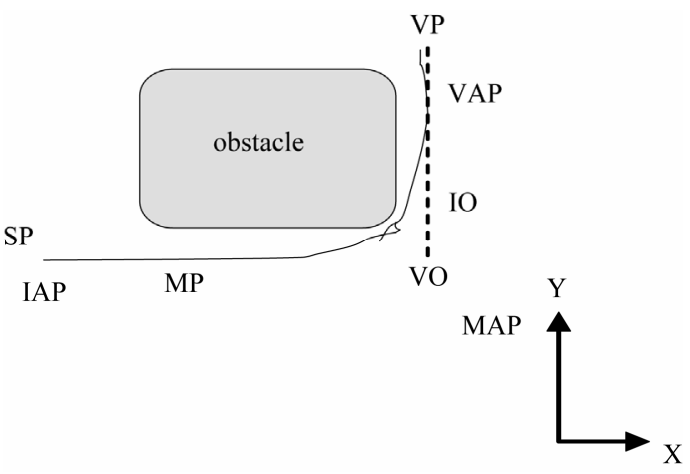

Figure 2. Robot obstacle avoidance.

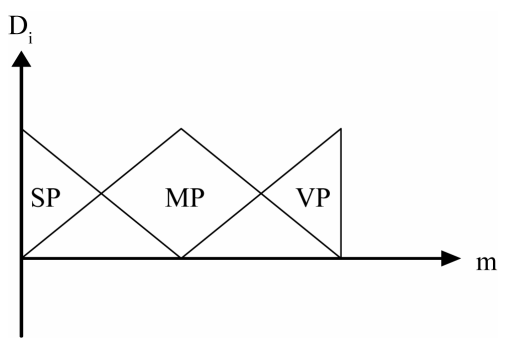

Figure 3. Memberships function of distance $D_{i}$.

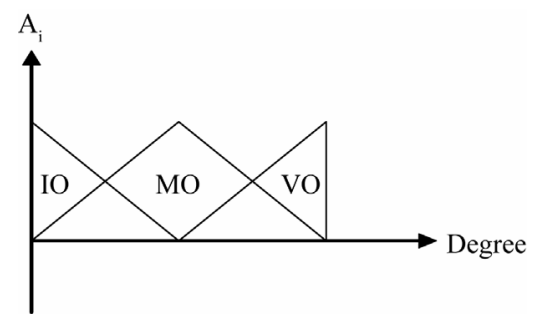

Figure 4. Memberships function of distance $A_{i}$.

- IO: Initial orientation.

- MO: Middle orientation.

- VO: Visual orientation.

The membership labels of distance $\mathrm{D}_{\mathrm{f}}$ are defined as (see the Figure 5):

- MO: Middle orientation.

- MO: Middle orientation.

- IAP: Initial arranged position.

- MAP: Middle arranged position.

- VAP: Visual arranged Position.

The membership functions of direction $\mathrm{A}_{\mathrm{f}}$ are presented in the Figure 6, where fuzzy labels are defined as:

- MP: Middle position.

- IAO: Initial arranged orientation.



Figure 5. Memberships function of distance $D_{f}$.

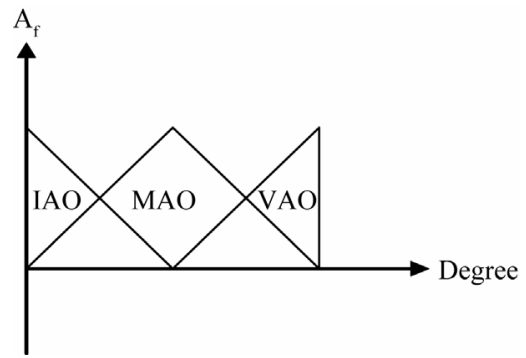

Figure 6. Memberships function of distance $A_{f}$.

- MAO: Middle arranged orientation.

- VAO: Visual arranged orientation.

The direction $A_{i}$ and $D_{i}$ are arranged at the end to get the best direction and position of avoiding the obstacle and attending target. The vehicle must learn to decided $A_{i}$ and $D_{i}$ using FL from a fuzzy linguistic formulation of human expert knowledge. This FL is trained to capture the fuzzy linguistic formulation of this expert knowledge is used and a set of rules are then established in the fuzzy rules as shown in Table 1.

The rules governing a fuzzy system are often written using linguistic expressions, which formalize the empirical rules by means of which a human operator is able to describe the process in question using his own experience. More, it is a way of linking input linguistic variables to the output ones.

If $\mathrm{x}$ and $\mathrm{y}$ are taken to be two linguistic variables, fuzzy logic allows these variables to be related by means of fuzzy conditional rules of the following type IF ( $\mathrm{x}$ is A) THEN ( $y$ is $B$ ). Where ( $x$ is $A$ ) is the premise of the rule, while (y is $\mathrm{B}$ ) is the conclusion. The premise defines the conditions in which the conclusions define the actions to be taken when the conditions of the premise are satisfied.

More specially, the degree of membership of the premise is calculated and through application of a fuzzy logic inference method to the conclusion, it allows the output $y$ to be determined. In general in a fuzzy conditional rule "if premise then conclusion" is made up of a statement in which fuzzy predicates $P_{j}$ of the general form are combined by different operators such as the fuzzy operators AND. 
Table 1. Rule infrence.

\begin{tabular}{ccc}
\hline \multirow{2}{*}{$\begin{array}{c}\text { Rule } \\
\text { inference }\end{array}$} & \multicolumn{2}{c}{ Table conditions } \\
\cline { 2 - 3 } & Statements & Result \\
\hline If $\left(\left(\mathrm{A}_{\mathrm{i}}\right.\right.$ is IAP $)$ and $\left(\mathrm{D}_{\mathrm{i}}\right.$ is $\left.\left.\mathrm{SP}\right)\right)$ & $\left(\left(\mathrm{D}_{\mathrm{f}}\right.\right.$ is $\left.\mathrm{AP}\right)$ and $\left(\mathrm{A}_{\mathrm{f}}\right.$ is IAO $\left.)\right)$ \\
If $\left(\left(\mathrm{A}_{\mathrm{i}}\right.\right.$ is $\left.\mathrm{MAP}\right)$ and $\left(\mathrm{D}_{\mathrm{i}}\right.$ is $\left.\left.\mathrm{MP}\right)\right)$ & $\left(\left(\mathrm{D}_{\mathrm{f}}\right.\right.$ is MAP $)$ and $\left(\mathrm{A}_{\mathrm{f}}\right.$ is $\left.\left.\mathrm{MAO}\right)\right)$ \\
If $\left(\left(\mathrm{A}_{\mathrm{i}}\right.\right.$ is $\left.\mathrm{VAP}\right)$ and $\left(\mathrm{D}_{\mathrm{i}}\right.$ is $\left.\left.\mathrm{VP}\right)\right)$ & $\left(\left(\mathrm{D}_{\mathrm{f}}\right.\right.$ is $\left.\mathrm{VAP}\right)$ and $\left(\mathrm{A}_{\mathrm{f}}\right.$ is $\left.\left.\mathrm{VAO}\right)\right)$ \\
\hline
\end{tabular}

To apply an inference method to the conclusion, it is first necessary to access the degree of membership of the premise, trough assessment of the degree of membership of each predicate $P_{j}$ in the premise. In our case, the principle of the technique consists in verifying for every degree membership functions.

Defuzzification is the output of the fuzzy system, it is a decision-making logic (written in a formula) adopted for the compute of the real value of the output. The final decision (defuzzification) is achieved to give the output of fuzzy controls and to converts the fuzzy output value produced by rules. The system must decide how to give the right output using fuzzy logic from a fuzzy linguistic formulation. The generation of rules operates itself according to the distribution of the training whole in fuzzy linguistic terms. The final decision (deffuzification) is accomplished to convert input of the fuzzy system after treatments with the inference rules.

The final decision (defuzzification) is achieved to give the output of fuzzy controls and to converts the fuzzy given by the principle of minimum and maximum to get from the start position (minimum principle) and the goal position (maximum principle).We calculate the centroid of the backend membership function correspond for each rule and we use the $\mathrm{U}$ factor of membership correspond for each rule.

This intelligent task uses the fuzzy linguistic terms and calculates for each degree of membership functions under expertise of an expert system ES. An ES is a computer program that functions, is in a narrow domain, dealing with specialized knowledge, generally possessed by human experts.

The expert system represents a good part of activities of the Artificial intelligence that makes call to knowledge on the domain treaty, these systems are capable to reach human expert performances for various types of tasks (diagnosis, conception in restraint domains). In our case, the principle of the technique consists in verifying for every degree membership function a whole of rules, or each rule is the shape: IF $<$ cond $>$ THEN $<$ name of the stain $>$, Where $<$ cond $>$ is a combination of predicates translating the spatial relations between the primitive of the unknown shape (if the logic used by the ES is the one of predicates.

\section{Simulation Results}

We denote that the configuration grid is a representation of the configuration space. In the configuration grid starting from any location to attend another one, cells are thus belonging to reachable or unreachable path.

Note that the set of reachable cells is a subset of the set of free configuration cells, the set of unreachable cell is a subset of the set of occupied configuration cells. By selecting a goal that lies within reachable space, we ensure that it will not be in collision and it exists some "feasible fuzzy path" such that the goal is reached in the environment. Having determined the reachability space, the algorithm works and operates on the reachability grid. This one specifies at the end the target area.

To execute the program, we have created several environments which contain many obstacles. The search area (environment) is divided into square grids. Each item in the array represents one of the squares on the grid, and its status is recorded as walkable or unwalkable area (obstacle). The robot starts from any position then using fuzzy logic learning must move and attends its target. The trajectory is designed in form of a grid-map, when it moves it must verify the adjacent case by avoiding the obstacle that can meet to reach the target at the same line.

As an example, the environment set up is shown in the Figure 7. The path is found by figuring all the fuzzy squares. Once the path is found, the robot moves from one square to the next until the target is reached, once we have simplified our search area into a convenient number of sub positions, as we have done with the grid design, the next step is to conduct a search to find the path. We do this by starting point, checking the adjacent squares, and training fuzzy model outward until we find our target. We start the search by the following steps: we have selected the starting position, it moves fuzzily forward as shown above in the Figure 8.

For unwalkable space, we compute the total size of free cells around danger (obstacle) area. This total may be at least greater or equal than to the length of architecture of robot. This is ensure the safety to our robot to not be in collision with the obstacle, and that the path $\mathrm{P}$ has enough security SE to attend it target where it is given by $\mathrm{P} \pm \mathrm{SE}$ ( $\mathrm{S}$ is size of security). For walkable space the robot reaches its target with the following steps illus- 




Figure 7. Fuzzy path environment set up 1.

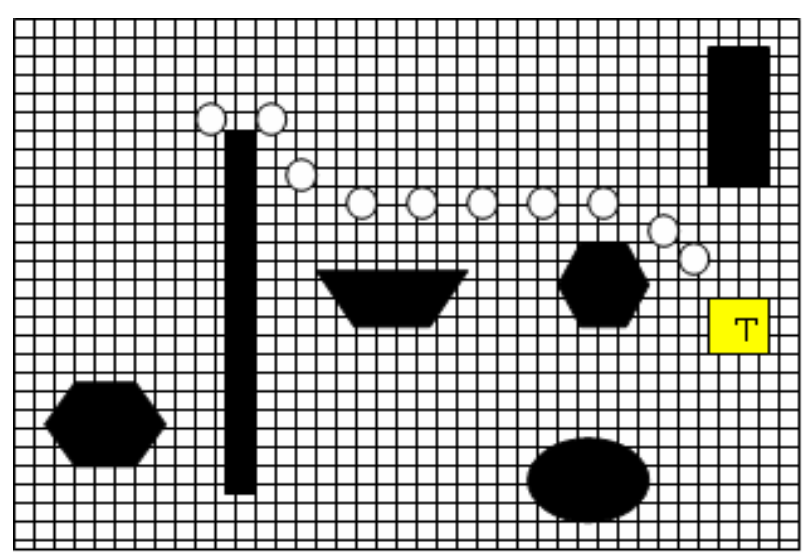

Figure 8. Fuzzy path environment set up 2.

trated in this algorithm:

Algorithm Of Work:

Begin

\section{Start set up environment Initialization}

SP

Move
IF $\{$ the obstacle is detected $\}$
DO
Begin
MP
IA
MAP
IAO
MAO

\section{L1: IF \{ the visual point is Reached? \}} DO
Begin
VP
VO
VAP

EAO
IF $\{$ The visual point is the target visual point?\}
DO
Begin
Target is attended.
End

Else GOTO L1.

\section{Conclusions}

The theory and practice of autonomous mobile robots are currently among the most intensively studied and promising areas in computer science and engineering which will certainly play a primary goal role in future. These theories and applications provide a source linking all fields in which intelligent control plays a dominant role. Cognition, perception, action, and learning are essential components of such-systems and their use is tending extensively towards challenging applications (service robots, micro-robots, bio-robots, guard robots, warehousing robots).

In this paper, we have presented a fuzzy logic implementation of navigation approach of an autonomous mobile robot in an unknown environment using hybrid intelligent control fuzzy logic and Expert system. Indeed, the main feature of FL combined with ES is the task fuzzy reasoning and inference capturing human expert knowledge to decide about the best avoidance direction getting a big safety of obstacle danger. Besides, the proposed approach can deal a wide number of environments. This system constitutes the knowledge bases of our approach allowing recognizing situation of the target localization and obstacle avoidance, respectively.

Also, the aim work has demonstrated the basic features of navigation of an autonomous mobile robot simulation. We have run our simulation in several environments where the robot succeeds to reach its target in each situation and avoids the obstacles capturing the behavior of intelligent expert system. The proposed approach can deal a wide number of environments. This navigation approach has an advantage of adaptivity such that the IAMR approach works perfectly even if an environment is unknown.

This proposed approach has made the robot able to achieve these tasks: avoid obstacles, deciding, perception, and recognition and to attend the target which are the main factors to be realized of autonomy requirements. Hence, the results are promising for next future work of this domain.

\section{REFERENCES}

[1] B. P. Gerkey and M. J. MatariĆ, "Principled Communication for Dynamic Multi-Robot Task Allocation, Expe- 
rimental Robotics VII, LNCIS 271," Springer-Verlag, Berlin, 2001, pp. 353-362.

[2] S. Saripalli, G. S. Sukhatme and J. F. Montgomery, "An Experimental Study of the Autonomous Helicopter Landing Problem," 8th International Symposium on Experimental Robotics, Sant'Angelo d'Ischia, 8-11 July 2002, pp. 8-11.

[3] T. Willeke, C. Kunz and I. Nourbakhsh, "The Personal Rover Project: The Comprehensive Design of a Dometic Personal Robot," Robotics and Autonomous Systems, Vol. 4, 2003, pp. 245-258.

[4] A. Howard, M. J. MatariĆ and G. S. Sukhatme, "An Incremental Self-Deployment Algorithm for Mobile Sensor Networks, Autonomous Robots," Special Issue on Intelligent Embedded Systems, Vol. 13, No. 2, 202, pp. 113126.

[5] B. P. Gerkey, M. J. MatariĆ and G. S. Sukhatme, "Exploiting Physical Dynamics for Concurrent Control of a Mobile Robot," Proceeding of the IEEE International Conference on Robotics and Automation (ICRA 2002), Washington DC, 11-15 May 2002, pp. 3467-3472.

[6] D. Estrin, D. Culler and K. Pister, PERVASIVE Computing IEEE, 2002, pp. 59-69.

[7] L. Moreno, E. A. Puente and M. A. Salichs, "World Modelling and Sensor Data Fusion in a Non Static Environment: Application to Mobile Robots," Proceeding of International IFAC Conference Intelligent Components and Instruments for Control Applications, Malaga, 20-22 May 1992, pp. 433-436.

[8] L. R. Medsker, "Hybrid Intelligent Systems," Kluwer Academic Publishers, 1995.

[9] O. Hachour and N. Mastorakis, "FPGA Implementation of Navigation Approach," WSEAS International Multiconference 4th WSEAS Robotics, Distance Learning and
Intelligent Communication Systems (ICRODIC 2004), Rio de Janeiro Brazil, 1-15 October, 2004, p. 2777.

[10] O. Hachour and N. Mastorakis, "Avoiding Obstacles Using FPGA-A New Solution and Application," 5th WSEAS International Conference on Automation \& Information (ICAI 2004), Venice, 15-17 November 2004, pp. 2827-2834.

[11] O. Hachour and N. Mastorakis, "Behaviour of Intelligent Autonomous Robotic Iar," Iasme Transaction, Vol. 1, No. 1, 2004, pp. 76-86.

[12] O. Hachour and N. Mastorakis, "Intelligent Control and Planning of IAR," 3rd WSEAS International Multiconfrence on System Science and Engineering, Rion De Janeiro, Brawil, 12-15 October 2004. http://www.wseas.org

[13] C. Kujawski, "Deciding the Behaviour of an Autonomous Mobile Road Vehicle," Proceeding of 2nd International IFAC Conference on Intelligent Autonomous Vehicles, Espoo, 1995, pp. 404-409.

[14] T. Willeke, C. Kunz and I. Nourbahsh, "The History of the Robot Museum Robot Series: An Evolutionary Study," American Association for Artificial Intelligence, 2001.

[15] W. Pedrycz, "Relevancy of Fuzzy Models," Information Sciences, Vol. 52, No. 3, 1990, pp. 285-302. doi:10.1016/0020-0255(90)90046-D

[16] K. Schilling and C. Jungius, "Mobile Robots for Planetary Explorations," Proceeding of 2nd International Conference IFAC, Intelligent Autonomous.

[17] S. Florczyk, "Robot Vision Video-Based Indoor Exploration with Autonomous and Mobile Robots," WILEYVCH Verlag GmbH \& Co. KGaA, Weinheim, 2005.

[18] O. Hachour and N. Mastorakis, "IAV: A VHDL Methodology for FPGA Implementation," WSEAS Transaction on Circuits and Systems, Vol. 3, No. 5, pp. 1091-1096. 